Vietnam Journal of Mechanics, VAST, Vol.43, No. 1 (2020), pp. 79 - 90

DOI: https://doi.org/10.15625/0866-7136/15554

\title{
EVALUATION OF THE EFFECT OF THE CONCENTRATION OF SEEDING PARTICLES ON SPIKE-EXCITATION DOPPLER UVP MEASUREMENT
}

\author{
Nguyen Tat Thang ${ }^{1, *}$ \\ ${ }^{1}$ Posts and Telecommunications Institute of Technology, Hanoi, Vietnam \\ *E-mail: thangnt@ptit.edu.vn
}

Received: 20 September 2020 / Published online: 21 December 2020

\begin{abstract}
In the study of fluid flows, one of the important parameters is the spatialtemporal velocity distribution. Experimental measurement of the parameter is required for the development and validation of various models in this field. Techniques for the measurement of flow velocity at single points have been in operation with great success for many years. However, there are situations where the measured data at one point is obviously not enough to understand structures in, e.g., turbulent/transient flows. One of the well-established and powerful methods for measuring velocity distribution is the UVP - Ultrasonic Velocity Profile method that enables measurements of the instantaneous velocity profile along a measurement line, i.e. the sound path. The new application of spike excitation along with the Doppler signal processing to the UVP method has recently been successfully tested. Regarding this new method, factors influencing the measurement result require further careful investigations. This study addresses, to some extent, the effect of the seeding-particle concentration on the results of spike-excitation UVP measurements. For the investigation, experimental measurements of water pipe flow have been carefully executed for a wide range of the particle concentration. The dependence of the measured data on the particle concentration is evaluated and reported. The result of this study suggests an appropriate range of the seeding-particle concentration in setting up spike-excitation UVP measurements.
\end{abstract}

Keywords: flow velocity measurement, velocity profile, UVP - Ultrasonic Velocity Profile, spike excitation, doppler signal processing.

\section{INTRODUCTION}

Single-phase liquid flow widely exists in industry, such as in the thermal hydraulic system of various power plants. The flow characteristics, flowrate etc. are mandatory for the proper and optimized operation of the system. Because, for example, they directly affect the heat transport and therefore the power generation, the plant safety etc.

The spatial-temporal distribution of the flow parameters is of great importance for detailed analyses of the flow dynamics. Besides, liquid velocity profiles can be used 
to calculate the flowrate, an important parameter for flow control. A high accuracy of the flowrate measurement can be derived. Therefore, the uncertainty of the flowrate measurement can be firmly decreased. Advanced techniques for flow measurement are under continuous improvement and development. Among them, the ultrasound techniques, e.g. the UVP method, to measure the velocity profile of single-phase liquid flow have been well established and of considerable interest.

The UVP method is a unique and powerful tool to measure the velocity profile of liquid flows. It exploits the ultrasonic echography and Doppler effect [1,2]. A UVP system comprises an ultrasonic sensor (for emitting and receiving ultrasound); a signal processing unit that includes a Pulser/Receiver $(\mathrm{P} / \mathrm{R})$, a highspeed digitizer, and signal processing electronics and/or software. In conventional/commercial UVP systems, the signal processing algorithm is usually based on the pulse-repetition Doppler-shift method. In contrast with the continuous-wave method, the pulsed-wave method enables velocity profile measurement [3]. The Doppler method uses the Doppler effect to calculate the fluid velocity. An important characteristic of the UVP method is that it is able to perform non-intrusive and online measurement of spatial-temporal velocity distribution. Measurement of opaque-liquid flows in non-transparent boundaries is possible, especially at critical industrial conditions, e.g. at very high temperature etc. In comparison, the optical PIV/PTV (i.e. Particle Imaging/Particle Tracking Velocimetry), and the Echo PIV (i.e. PIV that uses ultrasonic B-mode images) are also of particular interest [4,5]. However, measurement result is typically obtained from offline analyses because, presently, image processing is a time-consuming process, especially at high measurement resolution and/or image resolution [6]. The UVP method has found a wide range of applications including the flow measurement, flow monitoring etc. in laboratory, industry and engineering.

In the UVP method, the active element (usually the piezoelectric element) of the sensor emits ultrasonic pulses when it is excited by an electrical excitation pulse [2]. Conventionally, the Doppler method exploits either a constant-amplitude electrical pulse or a sinusoidal one to excite an ultrasonic sensor [3]. Such particular electrical excitation pulse is generated from specially designed pulser to generate electrical pulses that have a specific number of wave cycles and a required frequency. The design and implementation of the hardware of such pulser are usually complicated. These pulsers are mainly used for specific applications (e.g. in conventional UVP systems, medical ultrasound systems etc.) and not widely available for custom applications. In contrast, the electrical excitation pulse of a spike type (i.e. a very short-time voltage rise/fall) is most widely used, especially in the Non-Destructive Testing (NDT) industry. Such a pulse that is generated from spike pulsers has a wideband frequency spectrum in nature. Previously, in the UVP method, it was not used with the Doppler shift method but with wide-band signal processing methods, e.g. the Ultrasonic Time-Domain Correlation - UTDC [7] etc. The spike length is typically much shorter than that of the sinusoidal pulse. Hence, the spike pulse enables high-spatial-resolution measurements. Additionally, the spike pulser circuit has simple design and can be cheaply implemented $[8,9]$. Therefore, affordable spike pulsers can be widely found for custom applications. In the previous study [10], a UVP system that utilizes the electrical excitation pulse of the spike type has been successfully 
developed. Applications of the system to the measurements of both single-flows and two-phase flows present obvious potential of new UVP method (e.g. see [11,12]).

The conventional UVP measurement method that exploits sinusoidal-burst excitation UVP systems (both hardware and software: e.g. commercial UVP systems) has been well established. Thus, regarding the UVP measurement method that is based on the new spike-excitation UVP system, it is also of immense importance to carefully investigate the factors influencing the applicability, accuracy etc. of the method.

In UVP measurement, the seeding of the liquid flow is of critical importance since there must be enough reflected ultrasound so that the velocity profile of the flow can be measured. Several seeding methods are in use with the UVP method such as using seeding particles, generating gas bubbles etc. (e.g. see [13]). The first affecting factor that needs to be addressed is the concentration of the seeding ultrasonic reflectors. Here the particle concentration is defined as the number of ultrasonic reflectors per one measurement volume of the UVP method.

In the previous study [14], preliminary investigations and analyses have been carried out and briefly reported. Based on the received feedback about the received results presented, in this study, the effect of the seeding-particle concentration is fully investigated and analyzed experimentally. Measurements of instantaneous velocity profile of a liquid flow in a pipe have been carried out at varied seeding-particle concentration. Analyses of the measured velocity profile have been executed. The influence of the seeding-particle concentration on the velocity profile measured by the spike-excitation UVP system has been quantified. The suitable range of the seeding-particle concentration has been suggested. Accordingly, the received results towards these research objectives would make significant new contributions to in-depth clarifications of the spike excitation UVP method.

\section{EXPERIMENTAL SETUP}

\subsection{Flow loop}

A schematic drawing of the experimental flow loop is shown in Fig. 1. The loop consists of the following main parts:

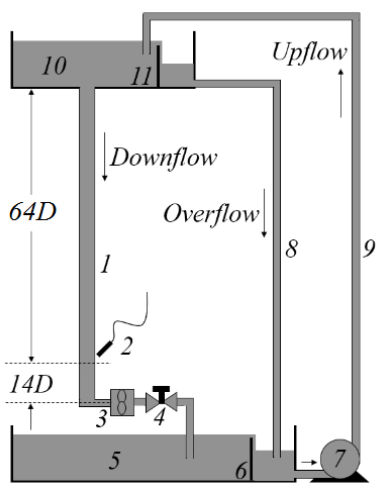

1- Test pipe (transparent acrylic, $50 \mathrm{~mm}$ inner diameter)

2- $8 \mathrm{MHz}$ ultrasonic sensor

3 - Turbine flowmeter (accuracy $\pm 2 \%$ )

4- Needle valve controlling the flowrate in the pipe 1

5- Floor tank

6- Overflow weir (in the floor tank)

7- Circulation pump

8- Overflow drain tube

9- Tube supplying water to the upper tank

10- Upper tank

11- Overflow weir (in the upper tank)

Fig. 1. Experimental flow loop [14] 
As shown in Fig. 1, the test flow is generated in the vertical pipe 1 . The sensor 2 is located at a distance of $64 \mathrm{D}$ from the pipe inlet and $14 \mathrm{D}$ from the end of the pipe (where $D$ is the inner diameter of the test pipe 1). $8 \mathrm{MHz}$ ultrasonic sensor 2 is used. The flowrate in the pipe 1 is measured by using the turbine flowmeter 3 . The needle valve 4 is used to control the flowrate of the test flow. Overflow weirs 6 and 11 are used to maintain constant water levels in the floor tank 5 and the upper tank 10 . Working liquid, tap water, is circulated through the up-flow pipe 9 to the upper tank by the pump 7 . The overflow from the upper tank returns to the floor tank through the pipe 8 . Nylon powder (WS-200P, Daicel Degussa Co., Ltd., Japan) is used as the seeding particles in the UVP measurements.

\subsection{Instrumentation}

The spike-excitation UVP system, both hardware and software, developed in [10] is used for the experimental investigation in this study. In general, the principle of UVP could be briefly explained as follows. An ultrasonic sensor is excited by electrical pulses from a pulser (i.e. a pulse generating circuit). Thus, the corresponding ultrasonic pulses generated from the sensor are transmitted along a measurement line, i.e. the sound path, through the tested flow. During the time between two consecutive emitted pulses, the sound reflected from the seeding particles along the line is received by the sensor itself and transmitted into a receiver (i.e. the receiver circuit). Since the sound speed is a known parameter, the received signal (i.e. a time series) can be exactly divided into segments that correspond to the equally-spaced positions along the sound path (i.e. the time-gating technique which is widely used in ultrasound systems, radar systems etc.). The Doppler shift frequency, hence the velocity, at each position can be calculated by using a Doppler-signal processing algorithm. Thus an instantaneous velocity profile can be obtained. The detailed principle of the pulsed-wave Doppler ultrasound and the signal processing methods can be found e.g. in [15-18].

In the UVP measurement of pipe flow, the ultrasonic sensor is typically set inclined an angle with respect to the flow direction so that the desired velocity profile can be always obtained. This is based on the fact that the Doppler effect, i.e. the change in the sound frequency, is measured for the movement towards or away from the sound source. The inclined angle is an input parameter and is selected based on detailed investigations prior to any UVP measurement [2].

The UVP system used in this investigation includes:

a) $8 \mathrm{MHz}$ ultrasonic sensor having active-element diameter $3 \mathrm{~mm}$ (Japan Probe Co. Ltd.).

b) Spike pulser and a signal receiver (JSR DPR300, JSR Ultrasonics Co., Ltd.) having maximum pulse-generating frequency $5000 \mathrm{~Hz}$.

c) PC with a high-speed digitizer having maximum sampling frequency $100 \mathrm{MHz}$ (NI PCI-5112, National Instrument Co. Ltd.) and signal processing software.

\subsection{Measurement setting}

Measurements were carried out in fully developed turbulent flow regime. The Reynolds number $R e$ was $11^{\prime} 000$ approximately where $R e$ is calculated by using Eqs. (1) 
and (2). The measurement conditions and UVP setting parameters are shown in Tab. 1.

$$
\begin{gathered}
R e=U D / \nu, \\
U=Q / A,
\end{gathered}
$$

where $U$ is the cross-sectional averaged velocity; $v$ is the kinematic viscosity of water; $Q$ is the flowrate in the pipe 1 ( $Q$ is measured by the flowmeter 3 ); $A$ is the cross-sectional area of the test pipe 1 (see Fig. 1).

Table 1. UVP settings and experimental conditions in the measurement of single-phase flow using the spike excitation UVP system

\begin{tabular}{lc}
\hline \multicolumn{1}{c}{ Parameter } & Value \\
\hline Inclined angle of the ultrasonic sensor $\left(^{\circ}\right)[2,19]$ & 45 \\
Number of instantaneous velocity profiles used to calculate an averaged profile $(-)$ & 1000 \\
Spatial resolution in the sound path $(\mathrm{mm})$ & 0.754 \\
Spatial resolution across the pipe $(\mathrm{mm})$ & 0.533 \\
Temporal resolution $(\mathrm{ms})$ & 51.2 \\
Temperature of the working liquid $\left({ }^{\circ} \mathrm{C}\right)$ & 30 \\
System pressure & Ambient pressure \\
\hline
\end{tabular}

The nylon powder used as the seeding particles in the measurements have an average diameter $80 \mu \mathrm{m}$, density $1.02 \mathrm{gram} / \mathrm{cm}^{3}$ that is almost the same as the water density. The particles are typically recommended for the UVP measurement of water flow [2]. In this study the definition of the particle concentration $C$ is as follows. $C$ is the spatially-averaged number of seeding particles in one UVP measurement volume (shown in Fig. 2). $C$ is determined once before each measurement by following the procedure

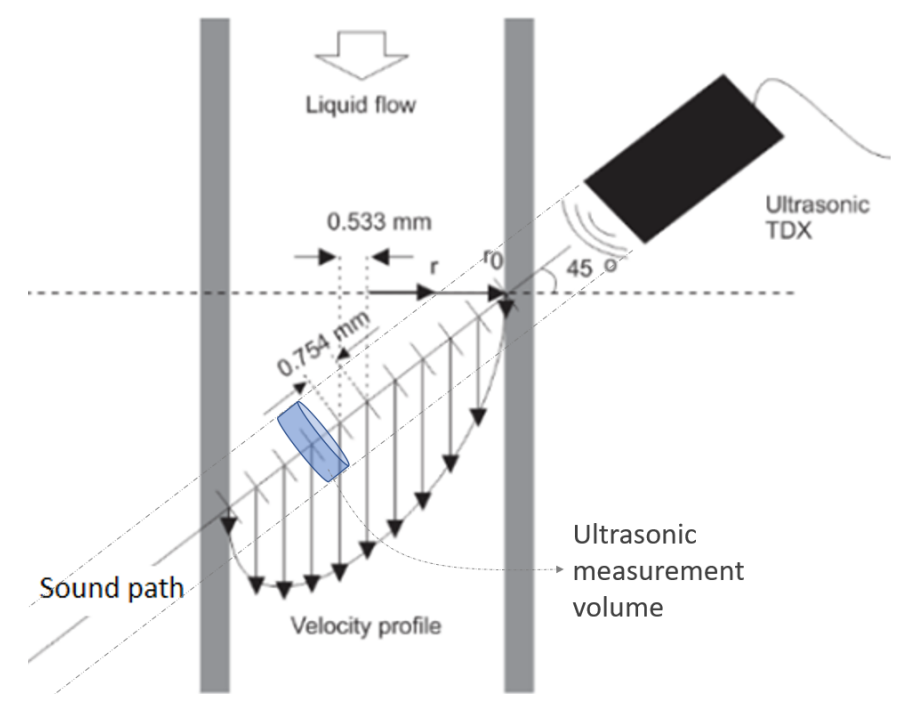

Fig. 2. Arrangement of the ultrasonic sensor and the settings of measurement parameters [20] 
shown next. First, the total volume of water used in the flow loop is converted into the total number of UVP measurement volumes by dividing the total water volume by the volume of one UVP measurement volume. Then, the particle concentration $C$ is calculated by dividing the total number of particles that are used in each experiment by the total number of UVP measurement volumes.

Measurements were carried out at the following conditions of the seeding particle concentration $C: 0.01,0.1,0.3,1,10,25,70$ and 100 (particles per UVP measurement volume). The settings of the ultrasonic sensor (TDX) are shown in Fig. 2.

The measurement volume of the UVP method is defined as the cylindrical volume surrounding a measurement position as shown in Fig. 2. Its thickness and diameter are specified by the spatial resolution and the ultrasonic beam diameter, respectively. The beam diameter is $3 \mathrm{~mm}$ approximately in this study.

\section{RESULTS AND DISCUSSION}

\subsection{Measured data}

Instantaneous velocity profiles of the flow are measured for different particle concentrations listed above. Then time-average velocity profiles are obtained by using the measured instantaneous velocity profiles. The average profiles and a profile obtained from the power law of turbulent pipe flow (i.e. Eq. (3) [21,22]) are plotted on the same graphs for each seeding particle concentration (Figs. 3-10). In the plots, the profiles are normalized by the measured velocity at the pipe center. The symbols are as follows: $r$ is the radius of the measurement positions along a velocity profile; $r_{0}$ is the inner radius of the pipe.

$$
u / U_{\max }=\left(1-r / r_{0}\right)^{1 / n}
$$

where $u$ is the flow velocity at radius $r$ (taken from the pipe center) along the profile; $U_{\max }$ is the velocity at the pipe center. In this study of turbulent pipe flow, the value $n=7$, which means the typical one-seventh power law velocity profile, is used.



Fig. 3. Time-average velocity profile (0.01 particles per measurement volume)

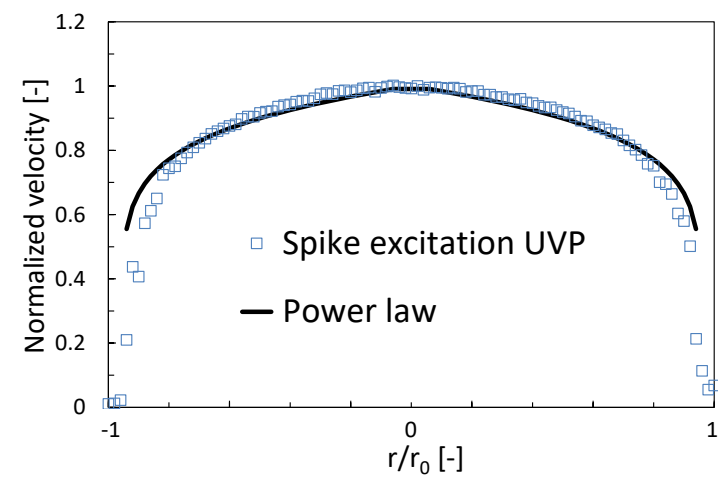

Fig. 4. Time-average velocity profile (0.1 particles per measurement volume) 


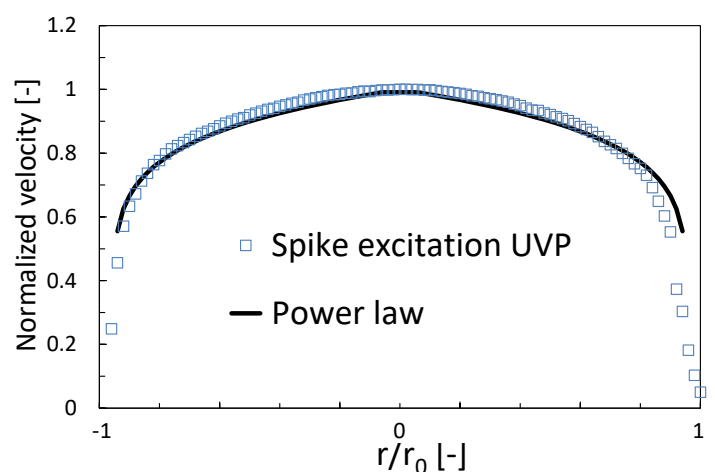

Fig. 5. Time-average velocity profile (0.3 particles per measurement volume)



Fig. 7. Time-average velocity profile (10 particles per measurement volume)



Fig. 9. Time-average velocity profile (70 particles per measurement volume)

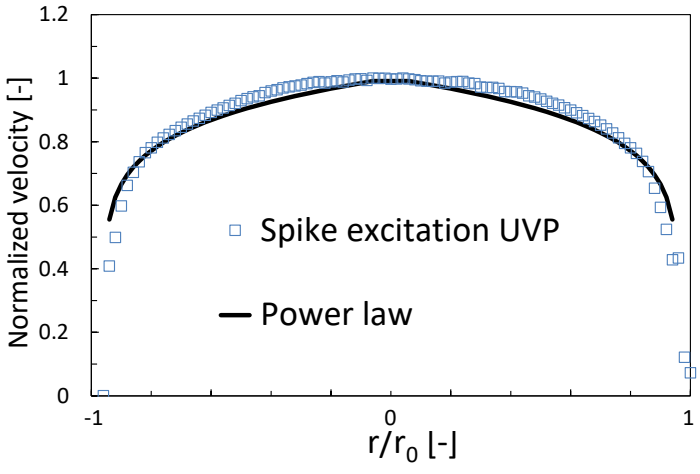

Fig. 6. Time-average velocity profile (1 particle per measurement volume)

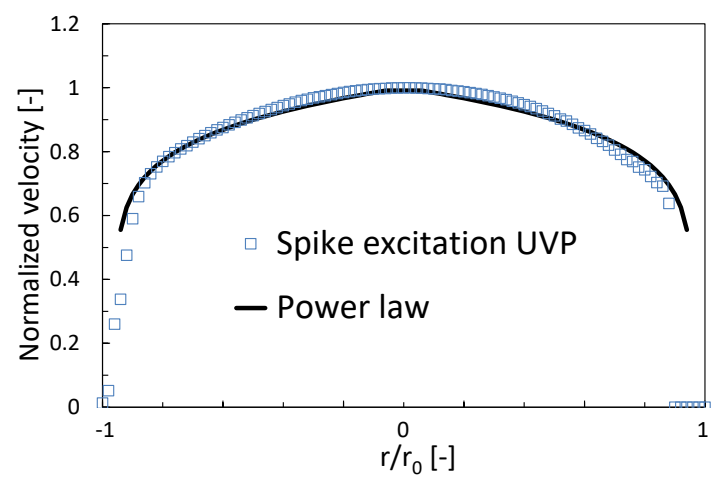

Fig. 8. Time-average velocity profile (25 particles per measurement volume)

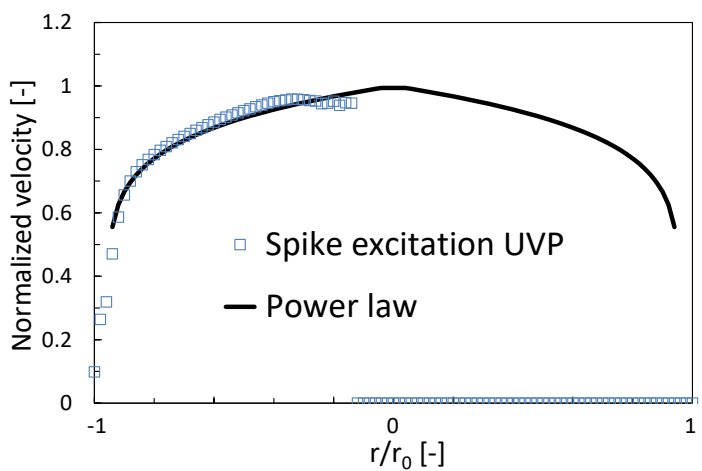

Fig. 10. Time-average velocity profile (100 particles per measurement volume) 
Nguyen Tat Thang

\subsection{Discussion}

As shown in Figs. 3 to 10, the spike-excitation UVP method can be applicable to a wide range of particle concentration. First, at very low particle concentration, i.e. $C=$ 0.01 (particle per measurement volume), the measured instantaneous velocity profile fluctuates. The backscattered ultrasound is weak, the SNR (Signal to Noise Ratio) is low, hence much noise presents in the measured data as what can be generally seen in the measurements using other UVP systems. Thus the averaged data are incorrect as shown in Fig. 3. It would be useful to keep in mind that, the values $C<1$ mean that, when they are written in the fractional form, i.e. $C=m / n$ where $m, n$ are integers $(m<n)$, statistically, there are $m$ particles distributed randomly in $n$ measurement volumes. Some volumes might not have any particles.

As $C$ increases, the SNR increases accordingly, the measured instantaneous velocity profiles improve. The averaged velocity profiles become better. They are in good agreement with that of the power law as seen in from Fig. $4(C=0.1)$ to $8(C=25)$. As general, closer to the sensor, the measured averaged velocity fits better to the theoretical profile since the SNR in such area is higher. Thus the corresponding instantaneous data should also be reliable. And the SNR in such range of $C$ and of the measurement depth (i.e. the distance along the sound path starting from the sensor surface) could be appropriate for the measurements, particularly in this investigation.

Since the UVP method is a non-intrusive and profile measurement method, the maximum measurable depth where the flow velocity can still be measured correctly is also a useful parameter. One of the main factors influencing the parameter is the particle concentration. As $C$ increases, the maximum measurable depth of the UVP method increases accordingly. However, this tendency is only applicable up to some value of the seedingparticle concentration. Up to some higher value, the maximum measurable depth decreases since the sound attenuation appears to play an important role when the particle concentration is high. Sound is attenuated strongly and its strength abruptly decreases at some distance from the sensor surface. The same problem applies to the backscattered ultrasound from the seeding particles. The sensor receives very weak signal returning from the flow field. Investigation of the phenomenon in the UVP method is also of interest.

As can be observed in Figs. 4 to 8 , in the far distance of the velocity profiles, the measured velocity first improves when $C$ increases from 0.1 to 1 (Figs. 4 to 6). The most appropriate velocity profile would be the one measured around $C=1$. Further increasing $C$, the measured data do not improve accordingly as can be seen in Figs. 7 and 8.

At increased $C$ (Figs. 9 and 10), the sound attenuation problem becomes obvious. The measured data are affected more strongly as $C$ increases. Beyond some critical depth, the measured velocity all becomes zero, but not noise as in the cases of low seeding particle concentration. It is useful to look at the received echo signal, i.e. the raw signal before applying signal processing, as briefly shown in Figs. 11(a) and 11(b). When $C=1$ (Fig. 11(a)), the echo signal distributes fairly along the measurement line. However, when $C=100$ (Fig. 11(b)), it is obvious that the signal strength is high in the close part of the profile but degenerates quickly in the far distance. 


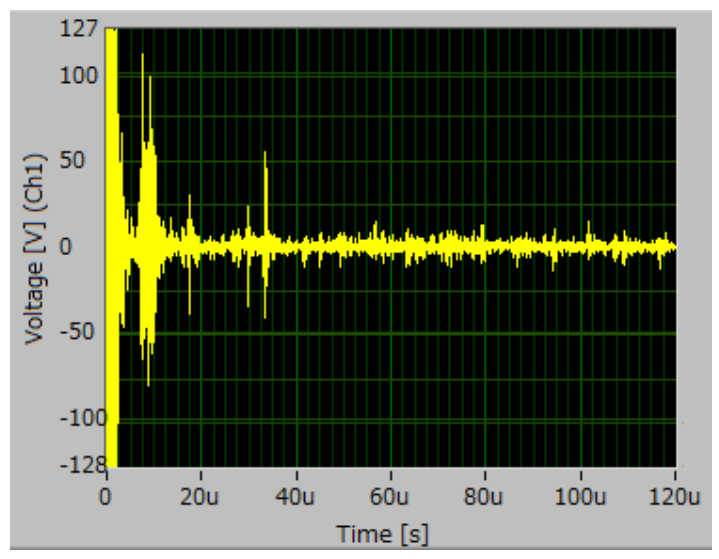

(a) 1 particle per measurement volume



(b) 100 particles per measurement volume

Fig. 11. Instantaneous received echo signal along the measurement line (raw data from the receiver output; $u$ : micro-second)

As generally expected, a UVP measurement should always be possible when $C=$ 1 (particle per measurement volume). Fig. 6 confirms that, when $C=1$, the averaged velocity profile measured by the spike-excitation UVP system appears to be the most reasonable one in comparison with the data of the power law of the pipe flow.

In addition, in order to confirm the accuracy of the measured data in this study, the measurement error needs to be quantified explicitly. In order to do so, the absolute error (in percentage) of the measurements is obtained by comparing the flowrate $Q$ calculated by using the measured velocity profile with that measured by the flowmeter 3 in Fig. 1) is shown in Tab. 2 for each particle concentration. To calculate the flowrate, the following discrete integral formular is used as shown in Eq. (4):

$$
Q=\frac{\pi}{3}\left\{\frac{r_{0}^{3}-r_{1}^{3}}{r_{0}-r_{1}} U_{0}+\sum_{i=0}^{k-2} \frac{r_{i+1}^{3}-r_{i+2}^{3}}{r_{i+1}-r_{i+2}}\left(U_{i+1}-U_{i}\right)+r_{k}^{2} U_{k}\right\},
$$

where $r_{0}=D / 2$ is the inner pipe radius; $U_{0}$ is the time-average velocity at the first measurement point from the pipe wall; $r_{i}$ is the radial distance from the pipe center to the measurement position $i ; U_{i}$ is the time-averaged velocity measured at the position $i$; $k$ is the total number of the measurement points along the radius of the pipe; $U_{k}$ is the time-averaged velocity at the pipe center (e.g. see [23-25]).

Table 2. Absolute error of the flowrate calculation

\begin{tabular}{lcccccccc}
\hline $\begin{array}{l}\text { Particle concentration C } \\
\text { (particles per measurement volume) }\end{array}$ & 0.01 & 0.1 & 0.3 & 1 & 10 & 25 & 70 & 100 \\
\hline $\begin{array}{l}\text { Particle concentration } \\
\text { (gram per flow volume in litter or } \mathrm{dm}^{3} \text { ) } \times 10^{2}\end{array}$ & 0.05 & 0.52 & 1.57 & 5.23 & 15.68 & 52.28 & 130.69 & 365.93 \\
\hline Measurement error $(\%)$ & 38.4 & 1.1 & 1.8 & 0.07 & 8 & 8.2 & 8.5 & $>8.6$ \\
\hline
\end{tabular}


The data of the measurement error further confirm that, the best error is obtained when $C=1$. Moreover, it is of significant interest to notice that, in this study on the spikeexcitation UVP method, the measured data (when $C$ is close to but less than 1 ) appear to have lower measurement error than those obtained when $C$ is above 1 . This characteristic is always preferable in UVP measurements since the lower the concentration of seeding particles, the lower the effects it might have on the flow dynamics.

\section{CONCLUDING REMARKS}

Investigation of the effects of the seeding particle concentration on the velocity profile measured by the spike-excitation UVP method has been systematically carried out experimentally for the first time. The following concluding remarks have been obtained:

Similar to the conventional UVP method, the spike excitation method works properly with the seeding particle concentration $C=1$ (particles in a measurement volume) in the measurement of turbulent single-phase pipe flow.

For the flow conditions in this study, when $C$ is too low $(C \sim 0.01$ or below), the measured instantaneous data would be all noise. The averaged data are incorrect.

In the range $0.1 \leq C \leq 10$, the measured data obtained are reliable. The averaged data have been compared with the theoretical ones. The accuracy of the measured data is firmly confirmed by further investigating the measurement error.

When $C$ increases ( $C \sim 25$ or above), the sound attenuation problem becomes significant that the measured data all become zero beyond some distance from the sensor surface. In these cases, the measurable depth is limited but the non-zero measured data can still be usable.

Since the seeding of the test flow is required in the UVP method, it is preferable to make the seeding particle concentration as low as possible to avoid any effects that the seeding particles might have on the flow dynamics. When the spike-excitation UVP method is used, particularly with the flow conditions investigated in this study, the favorable range of $C$ would be from around 0.1 to 1 .

In order to fully address the behavior of the spike excitation UVP system, further investigations are required for different flow conditions or types of flows etc.

\section{REFERENCES}

[1] Y. Takeda. Ultrasonic velocity profiler - from present to future. In Proceeding of the 5th International Symposium on Ultrasonic Doppler Method for Fluid Mechanics and Fluid Engineering, (2006), pp. 1-2.

[2] Y. Takeda. Ultrasonic Doppler velocity profiler for fluid flow. Springer Science \& Business Media, (2012).

[3] D. H. Evans and W. N. McDicken. Doppler ultrasound: physics, instrumentation and signal processing. John Wiley \& Sons, (2000).

[4] H. Zheng, L. Liu, L. Williams, J. R. Hertzberg, C. Lanning, and R. Shandas. Real time multicomponent echo particle image velocimetry technique for opaque flow imaging. Applied Physics Letters, 88, (26), (2006), p. 261915. https://doi.org/10.1063/1.2216875.

[5] F. Zhang, C. Lanning, L. Mazzaro, A. J. Barker, P. E. Gates, W. D. Strain, J. Fulford, O. E. Gosling, A. C. Shore, and N. G. Bellenger. In vitro and preliminary in vivo validation of echo 
particle image velocimetry in carotid vascular imaging. Ultrasound in Medicine $\mathcal{E}$ Biology, 37, (3), (2011), pp. 450-464. https://doi.org/10.1016/j.ultrasmedbio.2010.11.017.

[6] H. Yu, M. Leeser, G. Tadmor, and S. Siegel. Real-time particle image velocimetry for feedback loops using FPGA implementation. Journal of Aerospace Computing, Information, and Communication, 3, (2), (2006), pp. 52-62. https://doi.org/10.2514/1.18062.

[7] T. T. Nguyen, H. Kikura, N. H. Duong, H. Murakawa, and N. Tsuzuki. Measurements of single-phase and two-phase flows in a vertical pipe using ultrasonic pulse Doppler method and ultrasonic time-domain cross-correlation method. Vietnam Journal of Mechanics, 35, (3), (2013), pp. 239-256. https://doi.org/10.15625/0866-7136/35/3/3070.

[8] M. J. W. Povey. Ultrasonic techniques for fluids characterization. Elsevier, (1997).

[9] P. J. Shull. Nondestructive evaluation: theory, techniques, and applications. CRC Press, (2002).

[10] T. T. Nguyen. Study of ultrasonic velocity profiling method on boiling two-phase flow. PhD thesis, Tokyo Institute of Technology, Tokyo, Japan, (2016).

[11] T. T. Nguyen, N. Tsuzuki, H. Murakawa, N. H. Duong, and H. Kikura. Measurement of the condensation rate of vapor bubbles rising upward in subcooled water by using two ultrasonic frequencies. International Journal of Heat and Mass Transfer, 99, (2016), pp. 159-169. https://doi.org/10.1016/j.ijheatmasstransfer.2016.03.109.

[12] T. T. Nguyen, H. Kikura, H. Murakawa, and N. Tsuzuki. Measurement of bubbly two-phase flow in vertical pipe using multiwave ultrasonic pulsed Dopller method and wire mesh tomography. Energy Procedia, 71, (2015), pp. 337-351. https://doi.org/10.1016/j.egypro.2014.11.887.

[13] B. Birkhofer, T. Meile, G. De Cesare, S. A. K. Jeelani, and E. J. Windhab. Use of gas bubbles for ultrasound Doppler flow velocity profile measurement. Flow Measurement and Instrumentation, 52, (2016), pp. 233-239. https://doi.org/10.1016/j.flowmeasinst.2016.10.015.

[14] N. T. Thang. Two advanced non-intrusive methods for velocity distribution measurement in fluid mechanics with some recent research and development. In Proceedings of the International Conference of Fluid Machinery and Automation Systems - ICFMAS2018, (2018), pp. 653661.

[15] D. W. Baker. Pulsed ultrasonic Doppler blood-flow sensing. IEEE Transactions on Sonics and Ultrasonics, 17, (3), (1975), pp. 170-185.

[16] Y. Takeda. Velocity profile measurement by ultrasound Doppler shift method. International Journal of Heat and Fluid Flow, 7, (4), (1986), pp. 313-318. https://doi.org/10.1016/0142727x(86)90011-1.

[17] J. A. Jensen. Estimation of blood velocities using ultrasound: a signal processing approach. Cambridge University Press, (1996).

[18] T. T. Nguyen, H. Murakawa, N. Tsuzuki, H. N. Duong, and H. Kikura. Ultrasonic Doppler velocity profile measurement of single-and two-phase flows using spike excitation. Experimental Techniques, 40, (4), (2016), pp. 1235-1248. https://doi.org/10.1007/s40799-016-0123-8.

[19] H. Murakawa. Study on ultrasonic measurement technique for flow structure in bubbly flow. PhD thesis, Tokyo Institute of Technology, Tokyo, Japan, (2006).

[20] T. T. Nguyen, H. Murakawa, N. Tsuzuki, and H. Kikura. Development of multiwave method using ultrasonic pulse Doppler method for measuring two-phase flow. Journal of the Japanese Society for Experimental Mechanics, 13, (3), (2013), pp. 277-284. https://doi.org/10.11395/jjsem.13.277.

[21] P. K. Kundu, I. M. Cohen, and H. H. Hu. Fluid mechanics. Elsevier Academic Press, (2008). 
[22] L. J. De Chant. The venerable $1 / 7$ th power law turbulent velocity profile: a classical nonlinear boundary value problem solution and its relationship to stochastic processes. Applied Mathematics and Computation, 161, (2), (2005), pp. 463-474. https://doi.org/10.1016/j.amc.2003.12.109.

[23] S. Wada, H. Kikura, M. Aritomi, M. Mori, and Y. Takeda. Development of pulse ultrasonic Doppler method for flow rate measurement in power plant multilines flow rate measurement on metal pipe. Journal of Nuclear Science and Technology, 41, (3), (2004), pp. 339-346. https://doi.org/10.1080/18811248.2004.9715493.

[24] Y. Inoue, H. Kikura, H. Murakawa, M. Aritomi, and M. Mori. A study of ultrasonic propagation for ultrasonic flow rate measurement. Flow Measurement and Instrumentation, 19, (3-4), (2008), pp. 223-232. https://doi.org/10.1016/j.flowmeasinst.2007.06.013.

[25] W. Treenuson, N. Tsuzuki, H. Kikura, M. Aritomi, S. Wada, and K. Tezuka. Accurate flowrate measurement on the double bent pipe using ultrasonic velocity profile method. Journal of the Japanese Society for Experimental Mechanics, 13, (2), (2013), pp. 200-211. https://doi.org/10.11395/jjsem.13.200. 